\title{
ESTUDO DE UMA CONDIÇÃO PARA DETERMINAR A TRAJETÓRIA DE PRIMEIRO MOMENTO DOS SISTEMAS LINEARES SINGULARES SUJEITOS A SALTOS MARKOVIANOS
}

\author{
Amanda liz Pacífico Manfrim Perticarrari*, Andreia da Silva Meyer*, Nelson José \\ Peruzzi* $^{*}$, Ariadne Kaleda Marino ${ }^{\dagger}$, Mariana Zaneri Tavares ${ }^{\ddagger}$ \\ *Via de Acesso Prof.Paulo Donato Castellane s/n, 14884-900 \\ Depto de Ciências Exatas, FCAV, UNESP \\ Jaboticabal, SP, Brasil \\ $\dagger$ Via de Acesso Prof.Paulo Donato Castellane s/n, 14884-900 \\ Depto de Agronomia, FCAV, UNESP \\ Jaboticabal, SP, Brasil \\ $¥$ Via de Acesso Prof.Paulo Donato Castellane s/n, 14884-900 \\ Depto de Economia Rural, FCAV, UNESP \\ Jaboticabal, SP, Brasil
}

Emails: amanda@fcav.unesp.br, andreiameyer@fcav.unesp.br, peruzzi@fcav.unesp.br, ariadne.marino@gmail, mahzaneri@gmail.com

\begin{abstract}
This paper presents, from the study of the concept that guarantees solution of singular linear systems with Markovian jump parameters, a condition to determine the trajectory of the first moment that fits this class of systems.
\end{abstract}

Keywords - Stochastic dinamic, Markov chain, regularity.

Resumo - Este trabalho apresenta, a partir do estudo do conceito que garanta existência e unicidade de solução dos sistemas lineares singulares sujeitos a saltos Markovianos, denominado regularidade estocástica, uma condição necessária para determinar a trajetória de primeiro momento que se adequa a essa classe de sistemas.

Keywords - Dinâmica estocástica, cadeia de Markov, regularidade.

\section{Introdução}

Os sistemas singulares têm despertado interesse considerável na literatura devido ao fato desta classe ser apropriada para modelar sistemas que são muito utilizados em diversas áreas. Exemplos clássicos de aplicação são encontrados em modelagem de sistemas: aeronáuticos (Stevens and Lewis, 1991), de circuitos (Newcomb and Dziurla, 1989), econômicos (Luenberger and Arbel, 1977), interconectados em larga escala (Luenberger, 1978), robóticos (Lewis, 1986) e com processos químicos (Gilles, 1998).

Outra classe de sistemas que tem recebido grande atenção é a dos sistemas estocásticos, cuja evolução é influenciada por fatores aleatórios. Falhas, reparos em máquinas e modificações em parâmetros de sistemas são exemplos clássicos em que o uso exclusivo de argumentos determinísticos não é apropriado. Uma abordagem importante dessa classe de sistemas é baseada em modelos com saltos Markovianos nos parâmetros, que vem se tornando muito popular por possuírem propriedades eficientes para descrever este tipo de comportamento em sua dinâmica, veja (Costa et al., 2005), (Todorov and Fragoso, 2008), e as suas referências. Exemplos de aplicação podem ser encontrados em (do Val and Basar, 1999) e (Siqueira and Terra, 2004).
Ao incorporar saltos de Markov nos parâmetros de um sistema singular convencional, obtémse uma classe bastante ampla de sistemas, denominada de sistemas lineares singulares sujeitos a saltos Markovianos (SLSSM). Esta classe tem grande potencial de aplicações em sistemas físicos e econômicos, veja por exemplo (J. Lam and Boukas, 2007) e (Raouf and Boukas, 2007).

Considere o SLSSM em horizonte $T$ satisfazendo

$\Psi:\left\{\begin{array}{l}S_{\theta(k+1)} x(k+1)=F_{\theta(k)} x(k)+G_{\theta(k)} u(k), \\ 0 \leq k \leq T-1, \\ \theta(0)=\theta_{0}, \quad x(\ell)=x_{\ell}, \quad \ell \in \mathcal{L}\end{array}\right.$

para $k=0,1, \ldots$, sendo o par $(x(k), \theta(k))$ o estado do sistema, com $\theta \in \mathbb{T}=\{1, \ldots, N\}$, chamado de variável de salto ou modo e $x(k)$ a variável do sistema dinâmico associado a cada modo $\theta(k), y(k)$ a saída do sistema e $u(k)$ a entrada de controle do sistema. $\theta(k)$ é o estado de uma cadeia de Markov discreta no tempo com espaço de estado finito e com matriz de probabilidade de transição $\mathbb{P}=\left[p_{i j}\right], i, j=1, \ldots, N$, tal que $p_{i j}:=P(\theta(k+1)=j \mid \theta(k)=i)$ é a probabilidade do sistema passar do modo de operação $i$ para $j$; portanto $p_{i j} \geq 0$ deve ser satisfeita, para $i, j \in \mathbb{T}$ e, para cada $i, \sum_{j=1}^{N} p_{i j}=1$. Define-se a distribuição da cadeia de Markov, 
dada por $\pi_{i}(k)$, como $\pi_{i}(k)=P(\theta(k)=i)$ sempre que $i \in \mathbb{T}$. Sempre que $\theta(k)=i$ e $\theta(k+$ $1)=j, S_{\theta(k)}=S_{i}, F_{\theta(k)}=F_{i}, G_{\theta(k)}=G_{i} \mathrm{e}$ $H_{\theta(k)}=H_{i}$, sendo $S_{i}$ uma matriz singular, com $\operatorname{posto}\left(S_{i}\right)=r_{i} \leq n$. Considera-se os seguintes conjuntos de matrizes conhecidas de dimensões apropriadas $\mathrm{S}=\left(S_{1}, \ldots, S_{N}\right), \mathrm{F}=\left(F_{1}, \ldots, F_{N}\right)$ e $\mathrm{G}=\left(G_{1}, \ldots, G_{N}\right)$.

Observação 1. O conjunto $\mathcal{L}$ considerado em (1) fornece um modelo mais geral. Para recuperar a situação habitual, basta considerar $\mathcal{L}=$ $\{0\}$ e, desta maneira, esse conjunto define apenas a condição inicial $x(0)=x_{0}$. Também é notável que, no cenário sem saltos, $N=1$, a definição padrão de regularidade, que exige que $\operatorname{det}\left(\lambda S_{1}-F_{1}\right)$ não seja identicamente nulo, exceto para um número finito de $\lambda \in \mathbb{C}$, tem uma forte ligação da regularidade do sistema $\Psi$ com o conjunto $\mathcal{L}=\{0, N\}$

Esse trabalho desenvolveu-se a partir de uma revisão bibliográfica dos conceitos de SS, SLSM e SLSSM, com o objetivo de estabelecer uma condição para determinar a trajetória de primeiro momento que se adequa aos SLSSM a partir do estudo do conceito que garanta existência e unicidade de solução para essa classe de sistemas, denominado regularidade estocástica.

\subsection{Conceitos e resultados preliminares}

O conceito de regularidade estocástica para os SLSSM obtido através dos estudos de (Manfrim et al., 2008), (Manfrim, 2010), (Manfrim et al., 2011) e (Manfrim et al., 2012) é dado como segue.

Definição 1. Dizemos que o Sistema $\Psi$, ou equivalentemente $(S, \mathrm{~F}, \mathbb{P}, \mathcal{L})$, é regular estocasticamente no horizonte finito $T$ (ou $T$-regular) sempre que para cada sequência de controle $\mathcal{U}=$ $\{u(0), u(1), \ldots, u(T-1)\}$ e distribuição inicial da cadeia $\pi(0)$, existirem conjuntos $L_{m} \in \mathbb{R}$, $m \in \mathcal{L}$, tal que para cada realização da cadeia $\Theta(\omega)=\{\theta(0), \theta(1), \ldots, \theta(T-1)\}$, de probabilidade positiva, existir uma única trajetória $X=$ $\{x(0), x(1), \ldots, x(T)\}$ satisfazendo o sistema $\Psi$ e $x_{m} \in L_{m}$, com $m \in \mathcal{L}$.

Definição 2. Dizemos que o Sistema $\Psi$, ou equivalentemente $(S, \mathrm{~F}, \mathbb{P}, \mathcal{L})$, é regular estocasticamente quando o sistema $\Psi$ for $T$-regular para todo $T \geq 0$.

O próximo resultado mostra que a $T$ regularidade do Sistema $\Psi$ garante que os valores esperados envolvendo a trajetória (ou o processo) $\mathcal{X}=\{x(0), x(1), \ldots, x(T)\}$ estejam bem definidos.

Teorema 1. Seja $r$ um número inteiro positivo. Se o Sistema $\Psi$ é T-regular, então o processo $X$ é limitado quase certamente e tem momentos finitos de ordem $r$,

$$
\mathcal{E}\left\{\|(x(k))\|^{r}\right\} \leq\left\|L_{k}\right\|^{r},
$$

para algum $L_{k} \in \mathcal{R}^{n}$.

Uma vez que o Sistema $\Psi$ possui todos seus momentos bem definidos, podemos empregar o Teorema Central do Limite para fazer uma aproximação destes momentos, pois, como veremos adiante, o cálculo direto pode ser uma tarefa difícil. O próximo resultado é uma extensão direta dos resultados apresentados em (James, 1996), desta maneira a prova é omitida.

Proposição 1. Considere o Sistema $\Psi$ T-regular. Se $0 \leq\|(x(k))\|^{r} \leq\left\|L_{k}\right\|^{r}$, então

$$
\operatorname{Var}\left(\|(x(k))\|^{r}\right) \leq \frac{\left\|L_{k}\right\|^{2 r}}{4} .
$$

Teorema 2. Considere o Sistema $\Psi$ T-regular. Sejam $\mu_{k}=\mathcal{E}\left\{\|x(k)\|^{r}\right\}$, sendo $r$ um inteiro positivo, $\bar{X}_{k, M}$ a média para uma amostra de tamanho $M$, ou seja,

$$
\bar{X}_{k, M}=\frac{\sum_{b=1}^{M}\left\|x\left(k, w_{b}\right)\right\|^{r}}{M},
$$

e $\Sigma_{k, M}=\operatorname{Var}\left(\bar{X}_{k, M}\right)$ então existe $L_{k}$ tal que $\Sigma_{k, M} \leq \frac{\left\|L_{k}\right\|^{2 r}}{4 M}$, e a distribuição de $\bar{X}_{k, M}$ converge para $N\left(\mu_{k}, \Sigma_{k, M}\right)$, quando $M$ tende a infinito. Em particular, $\bar{X}_{k, M}$ converge em probabilidade para $\mu_{k}$.

Observação 2. Se o Sistema $\Psi$ é T-regular, através do Teorema 1 sabemos que $\mathcal{E}\{x(k)\}$ é bem definido para todo $0 \leq k \leq T$. Utilizando o Teorema da Probabilidade Total podemos desenvolver expressões como abaixo, que serão úteis ao longo do texto.

$$
\begin{aligned}
& \mathcal{E}\{x(k)\}=\sum_{i=1}^{N} \mathcal{E}\left\{x(k) 1_{\{\theta(k)=i\}}\right\} \\
& \mathcal{E}\{x(k)\}=\sum_{j=1}^{N} \sum_{i=1}^{N} \mathcal{E}\left\{x(k) 1_{\{\theta(k)=i, \theta(k+1)=j\}}\right\} .
\end{aligned}
$$

\subsection{Resultados}

A partir dos resultados apresentados no Teorema 1 e Observação 2, temos uma condição de primeiro momento para o Sistema $\Psi$ e com isso apresentamos uma condição necessária testável para a $T$ regularidade no Teorema 3 adiante. Outras podem ser obtidas através de diversas relações de equivalência para $\mathcal{E}\{x(k)\}$ como em (3).

Lema 1. Considere o Sistema $\Psi$ T-regular, com U e $x(0)$ fixos. Sempre que

$$
q_{i}(k):=\mathcal{E}\left\{x(k) 1_{\{\theta(k)=i\}}\right\},
$$


então $q_{i}(k)$ satisfaz

$$
\begin{aligned}
S q_{j}(k+1)= & \sum_{i=1}^{N} p_{i j} F_{i} q_{i}(k)+ \\
& +\sum_{i=1}^{N} p_{i j} \pi_{i}(k) G_{i} u(k), j \in \mathbb{T} .
\end{aligned}
$$

Proof: Pré multiplicando (1) por $1_{\{\theta(k+1)=j\}}$ temos que

$$
\begin{aligned}
& S x(k+1) 1_{\{\theta(k+1)=j\}}= \\
& =F_{\theta(k)} x(k) 1_{\{\theta(k+1)=j\}}+ \\
& +G_{\theta(k)} u(k) 1_{\{\theta(k+1)=j\}} .
\end{aligned}
$$

Aplicando o valor esperado em ambos os lados da igualdade (5) obtemos:

$$
\begin{aligned}
& \mathcal{E}\left\{S x(k+1) 1_{\{\theta(k+1)=j\}}\right\}= \\
&= \mathcal{E}\left\{F_{\theta(k)} x(k) 1_{\{\theta(k+1)=j\}}\right\}+ \\
&+\mathcal{E}\left\{G_{\theta(k)} u(k) 1_{\{\theta(k+1)=j\}}\right\} .
\end{aligned}
$$

Utilizando a seguinte propriedade: "Seja $A$ uma variável aleatória, então

$$
\mathcal{E}\{A\}=\sum_{i=1}^{N} \mathcal{E}\left\{A 1_{\{\theta(k)=i\}}\right\} ”
$$

podemos reescrever (6) como

$$
\begin{aligned}
S \mathcal{E}\{ & \left.x(k+1) 1_{\{\theta(k+1)=j\}}\right\}= \\
= & \sum_{i=1}^{N} \mathcal{E}\left\{F_{\theta(k)} x(k) 1_{\{\theta(k+1)=j\}} 1_{\{\theta(k)=i\}}\right\}+ \\
& +\sum_{i=1}^{N} \mathcal{E}\left\{G_{\theta(k)} u(k) 1_{\{\theta(k+1)=j\}} 1_{\{\theta(k)=i\}}\right\},
\end{aligned}
$$

ou seja,

$$
\begin{aligned}
S \mathcal{E} & \left\{x(k+1) 1_{\{\theta(k+1)=j\}}\right\}= \\
= & \sum_{i=1}^{N} F_{i} \mathcal{E}\left\{x(k) 1_{\{\theta(k+1)=j, \theta(k)=i\}}\right\}+ \\
& +\sum_{i=1}^{N} G_{i} \mathcal{E}\left\{u(k) 1_{\{\theta(k+1)=j, \theta(k)=i\}}\right\} .
\end{aligned}
$$

Utilizando a seguinte propriedade

$$
\mathcal{E}\left\{Z_{w} 1_{\{w=i\}}\right\}=\mathcal{E}\left\{Z_{w} \mid w=i\right\} P(w=i)
$$

podemos reescrever (7) como

$$
\begin{aligned}
& S \mathcal{E}\left\{x(k+1) 1_{\{\theta(k+1)=j\}}\right\}= \\
& =\sum_{i=1}^{N} p_{i j} F_{i} \mathcal{E}\left\{x(k) 1_{\{\theta(k)=i\}}\right\}+ \\
& \quad+\sum_{i=1}^{N} p_{i j} G_{i} \mathcal{E}\left\{u(k) 1_{\{\theta(k)=i\}}\right\} .
\end{aligned}
$$

Sabendo que $u(k)$ é independente de $1_{\{\theta(k)=i\}}$ temos que

$$
\begin{aligned}
& \mathcal{E}\left\{u(k) 1_{\{\theta(k)=i\}}\right\}= \\
& \quad=\mathcal{E}\{u(k)\} \mathcal{E}\left\{1_{\{\theta(k)=i\}}\right\} \\
& =u(k) P(\theta(k)=i) \\
& =\pi_{i}(k) u(k),
\end{aligned}
$$

assim podemos reescrever (8) como

$$
\begin{aligned}
& S \mathcal{E}\left\{x(k+1) 1_{\{\theta(k+1)=j\}}\right\}= \\
& =\sum_{i=1}^{N} p_{i j} F_{i} \mathcal{E}\left\{x(k) 1_{\{\theta(k)=i\}}\right\}+ \\
& \quad+\sum_{i=1}^{N} p_{i j} \pi_{i}(k) G_{i} u(k) .
\end{aligned}
$$

Definindo $q_{i}(k):=\mathcal{E}\left\{x(k) 1_{\{\theta(k)=i\}}\right\}$, podemos reescrever (10) como

$$
\begin{aligned}
S q_{j}(k+1)=\sum_{i=1}^{N} p_{i j} F_{i} q_{i}(k)+ & \\
& +\sum_{i=1}^{N} p_{i j} \pi_{i}(k) G_{i} u(k) .
\end{aligned}
$$

O Lema 1 nos proporciona uma condição necessária testável para a $T$-regularidade, como destacamos no próximo resultado.

Teorema 3. Se o Sistema $\Psi$ é T-regular então existe solução para o Sistema (4).

Proof: Uma vez que o Sistema $\Psi$ é $T$-regular e $q_{i}(k)=\mathcal{E}\left\{x(k) 1_{\{\theta(k)=i\}}\right\}$, aplicando o Lema 1 segue o resultado.

\subsection{Conclusão}

Nesse trabalho foi estudado o conceito de regularidade estocástica de horizonte finito e regularidade estocástica. A partir desse estudo, foi possível verificar que esses conceitos garantem que os valores esperados envolvendo a trajetório dos SLSSM proposto estão bem definidos. Dessa maneira apresentamos uma condição necessária testável para a verificação da $T$-regularidade envolvendo o primeiro momento dessa classe de sistemas.

\section{Referências}

Costa, O. L. V., Fragoso, M. D. and Marques, R. P. (2005). Discrete-time Markov jump linear systems: Probability and its applications, Springer-Verlag, London.

do Val, J. B. R. and Basar, T. (1999). Receding horizon control of jump linear systems and a macroeconomic policy problem, Journal of Economic Dynamics \& Control 23: 10991131. DOI: 10.1016/S0165-1889(98)00058-X 
Gilles, E. D. (1998). Network theory for chemical processes, Chemical Engineering and Tech-nology 21(2): 121-132. DOI: 10.1002/ (SICI)1521-4125(199802)21:2<211::AIDCEAT121>3.0.CO;2-U

J. Lam, Z. Shu, S. X. and Boukas, E. K. (2007). Robust $H_{\infty}$ control of descriptor discretetime Markovian jump systems, International Journal of Control 80(3): $374-385$. DOI: 10.1080/00207170600999322

James, B. (1996). Probabilidade: Um curso em nível intermediário., Vol. 2, IMPA.

Lewis, F. L. (1986). A survey of linear singular systems, Circuits, Systems, and Signal Pro-cessing 5(1): 3-36.

DOI: $10.1007 / \mathrm{BF} 01600184$

Luenberger, D. G. (1978). Time-invariant descriptor systems, Automatica 14(5): $473-480$. DOI: $10.1016 / 0005-1098(78) 90006-7$

Luenberger, D. G. and Arbel, A. (1977). Singular dynamic Leontief systems, Econometrica 45(4): 991-995. DOI: 10.2307/1912686

Manfrim, A. L. P. (2010). Sistemas lineares singulares sujeitos a saltos Markovianos, Tese de Doutorado, Universidade de São Paulo .

Manfrim, A. L. P., Costa, E. F. and Terra, M. H. (2011). Condição necessária para a existência de solução dos sistemas lineares singulares sujeitos a saltos Markovianos, Anais do I Congresso de Matemática Aplicada e Computacional da Região Sudeste pp. 463-467.

Manfrim, A. L. P., Peruzzi, N. J., Meyer, A. S., Tavares, M. Z. and Marino, A. K. (2012). Estudo da regularidade estocástica dos sistemas lineares sujeitos a saltos Markovianos, Anais do Congresso de Matemática Aplicada e Computacional - CMAC Nordeste 2012 pp. $316-319$.

Manfrim, A. L. P., Terra, M. H., Costa, E. F. and Ishihara, J. Y. (2008). On the regularity for singular linear system with Markov jump parameters, Proceedings of the 17th World Congress of the International Federation of Automatic Control pp. 9987-9992.

Newcomb, R. W. and Dziurla, B. (1989). Some circuits and systems applications of semistate theory, Journal Circuits, Systems, and Signal Processing 8(3): 235-260.

DOI: $10.1007 / \mathrm{BF} 01598413$

Raouf, J. and Boukas, E. (2007). Stabilization of discontinuous singular systems with Markovian switching and saturating inputs, number 1, pp. 2442-2447.

Siqueira, A. A. G. and Terra, M. H. (2004). Nonlinear and Markovian H-infinity controls of underactuated manipulators, IEEE Transactions on Control System Technology 12(6): 811-826.

DOI: 10.1109/TCST.2004.833626
Stevens, B. L. and Lewis, F. L. (1991). Aircraft Modeling, Dynamics and Control, Wiley, New York.

Todorov, M. G. and Fragoso, M. D. (2008). Output feedback H-infinity control of continuoustime infinite Markovian jump linear systems via LMI methods, SIAM Journal on Control and Optimization 47(2): 950-974.

DOI: $10.1137 / 060675162$ 